\title{
UK Renal Registry 11th Annual Report (December 2008): Chapter 13 Demography of the UK paediatric Renal Replacement Therapy population
}

\author{
Malcolm A Lewis ${ }^{a}$, Joanne Shaw ${ }^{a}$, Manish Sinha ${ }^{b}$, Shazia Adalat ${ }^{b}$, Farida Hussain $^{c}$ and \\ Carol Inward ${ }^{\text {d }}$ \\ ${ }^{a}$ Manchester Childrens Hospital, Manchester, UK; ${ }^{b}$ Evelina Childrens Hospital, London, UK; ${ }^{c}$ Nottingham University \\ Hospital, Nottingham, UK; ${ }^{d}$ Bristol Royal Hospital for Children, Bristol, UK
}

\section{Key Words}

Aetiology · Children · Demography · ESRD · Established renal failure $\cdot$ Incidence $\cdot$ Paediatric $\cdot$ Prevalence $\cdot$ Survival

\begin{abstract}
Aims: To describe the demographics of the paediatric RRT population in the UK and analyse changes in demographics with time. Methods: Extraction and analysis of data from the UK paediatric Renal Registry. Results: The UK paediatric established renal failure (ERF) population in April 2008 was 875 patients. The prevalence under the age of 16 years was 55 per million age related population ( $\mathrm{pmp}$ ) and the incidence $7.92 \mathrm{pmp}$. The incidence and prevalence for South Asian and Other ethnic groups were 3 times that of the White and Black populations. Renal dysplasia was the most common cause of ERF accounting for $33 \%$ of prevalent cases. Diseases with autosomal recessive inheritance were more common in patients from ethnic minority groups. The spectrum of diseases seen has changed over a generation. Overall 5 year survival for children with ERF was $91.8 \%$. Five year survival of infants starting dialysis was just $62 \%$. Transplanted patients accounted for $74 \%$ of the current population. The proportion with grafts from living donors has steadily risen to $34 \%$. Children from
\end{abstract}

(C) 2009 S. Karger AG, Basel

$1160-2110 / 09 / 1115-0257 \$ 26.00 / 0$

Fax +4161306 1234 E-Mail karger@karger.ch www.karger.com
Accessible online at: www.karger.com/nec ethnic minority groups were less likely to have an allograft and living donation was less frequent in this population. For those on dialysis, $57 \%$ were receiving peritoneal dialysis. This was the main treatment modality for patients under 4 years of age. Conclusions: The paediatric ERF population continued to expand slowly. Incidence and prevalence rates were stable and similar to other developed nations. The high incidence in patients from ethnic minority groups will lead to a greater proportion of the population being from these groups in time. To maintain the high proportion of engrafted patients it will be necessary to encourage living donation in the ethnic minority population. The spectrum of diseases seen has already changed over a generation with the treatment of young children with diseases such as congenital nephrosis. The incidence of cystinosis causing ERF was reduced, probably reflecting better early treatment.

\section{Introduction}

Knowledge of the demography of a patient population is essential for the planning of services and the assessment of outcomes. Within the UK, treatment of 
paediatric patients with established renal failure (ERF) takes place within 13 regional centres (Scotland 1, Wales 1, Northern Ireland 1 and England 10). All centres have facilities for peritoneal dialysis and haemodialysis. Ten of the 13 centres undertake transplantation for children. As part of the development of a national Renal Registry the British Association for Paediatric Nephrology (BAPN) began collecting data on children in 1996. The aim has been to provide a fully integrated data collection programme which would seamlessly merge with the adult data in the UK Renal Registry. Some data is also available from BAPN national audits undertaken in years before the inception of the Paediatric Registry. Whilst the completeness of data collection has varied over the years an attempt was made in April 2008 to capture the whole of the population of children with ERF being treated in centres across the UK for these analyses.

The term ERF used within this chapter is synonymous with the terms of End Stage Renal Failure (ESRF) and End Stage Renal Disease (ESRD) which are in more widespread international usage. Within the UK, patient groups have disliked the term 'End Stage' which formerly reflected the inevitable outcome of this disease.

\section{Methods}

Data collection took place across the UK looking at patient status on 1st April 2008. Some centres collected data electronically and used the data transfer channel to the UK Renal Registry for data transfer. Other centres used paper data collections which were then manually input into the current paediatric registry database. Whilst extensive demographic details were available from 12 centres, the smallest centre (Southampton) was on this occasion only able to provide a total patient number and treatment modality for each patient. Data were then extracted and analysed using Microsoft Excel and statistical analysis was performed using the StatsDirect programme and Fisher's exact test.

\section{Results}

The UK paediatric ERF population

The UK paediatric ERF population on 1st April 2008 was 875 patients. One centre of 22 patients could not provide further demographic data. The age and gender distribution of the remaining 853 patients is shown in table 13.1. Overall the gender ratio of males to females was just over
Table 13.1. The UK paediatric ERF population on 1st April 2008, by age and gender

\begin{tabular}{lcrrrr}
\hline & Patients & Male & Female & Ratio & \% total \\
\hline White & 664 & 415 & 249 & $1.67: 1$ & 77.8 \\
S Asian & 144 & 73 & 68 & $1.07: 1$ & 16.9 \\
Black & 23 & 16 & 7 & $2.29: 1$ & 2.7 \\
Other & 22 & 10 & 12 & $0.83: 1$ & 2.6 \\
Total & $\mathbf{8 5 3}$ & $\mathbf{5 1 4}$ & $\mathbf{3 3 6}$ & $\mathbf{1 . 5 3 : 1}$ & $\mathbf{1 0 0 . 0}$ \\
$<18$ years & 812 & 491 & 318 & $1.54: 1$ & 95.2 \\
$<15$ years & 536 & 327 & 207 & $1.58: 1$ & 62.8 \\
\hline
\end{tabular}

*gender unknown for 3 patients

1.5 to 1 . Ethnic minority groups composed just over $22 \%$ of the population.

Using previous BAPN audits in 1986 and 1992, together with subsequent data from the UK Paediatric Registry it was possible to look at the growth of the paediatric ERF population. To allow direct comparison, these data only included those under the age of 15 years and are shown in figure 13.1. The data point for 2008 assumed the proportion of the patients under 15 years of age at Southampton was the same as for the rest of the country. The confidence limits for this number are \pm 7 . Table 13.2 shows a more detailed breakdown of the ERF population according to age. For this analysis the 22 patients for Southampton were excluded. Whilst the total number of patients being treated across the UK continued to gradually increase with time, the proportion under the age of 15 years seems to be on a plateau.

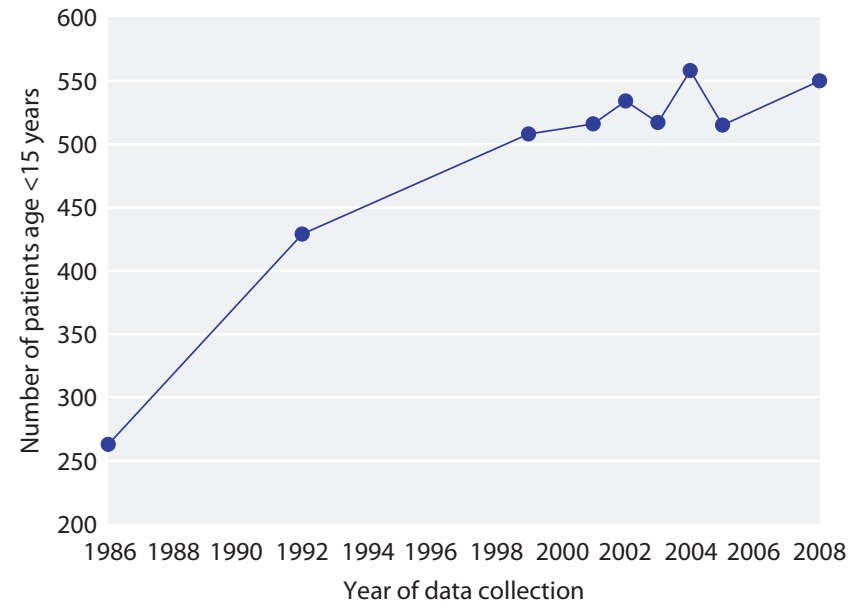

Fig. 13.1. Prevalent patients below 15 years of age on RRT in the UK 
Table 13.2. Prevalent paediatric ERF population by age and year of data collection

\begin{tabular}{|c|c|c|c|c|c|c|c|c|c|}
\hline \multirow[b]{2}{*}{ Age group (yrs) } & \multicolumn{9}{|c|}{ Patient population data for the years of (on 1st April) } \\
\hline & 1986 & 1992 & 1999 & 2001 & 2002 & 2003 & 2004 & 2005 & 2008 \\
\hline $2-4.9$ & & 55 & 46 & 56 & 58 & 56 & 51 & 45 & 68 \\
\hline 5-9.9 & & 150 & 151 & 146 & 147 & 141 & 166 & 157 & 148 \\
\hline $10-14.9$ & & 208 & 293 & 301 & 315 & 310 & 329 & 299 & 298 \\
\hline Total $<20$ & & & 761 & 790 & 793 & 773 & 802 & 768 & 851 \\
\hline
\end{tabular}

The proportion of ethnic minority (EM) patients has increased and when compared to the previous most complete data collection in 2004, this increase was significant $(\mathrm{p}=0.0078)$. These data are shown in figure 13.2.

All patients under the age of 16 years in the UK are managed by paediatric centres. To allow meaningful comparisons and equal age distributions, patients were divided into four year age bands from birth to 20 years. These data are shown in table 13.3 for the years of 2002, 2003, 2004 and 2005 when analyses were undertaken together with the data from the current analysis. Across all years, there was a rise in numbers with each increase in age band until the 16 to 20 year band when the population falls due to transfers to adult centres. In the current dataset the number of patients below the age of 4 years has risen and compared with the 2004 data the proportion under the age of 4 years is significantly larger $(\mathrm{p}=0.0175)$.

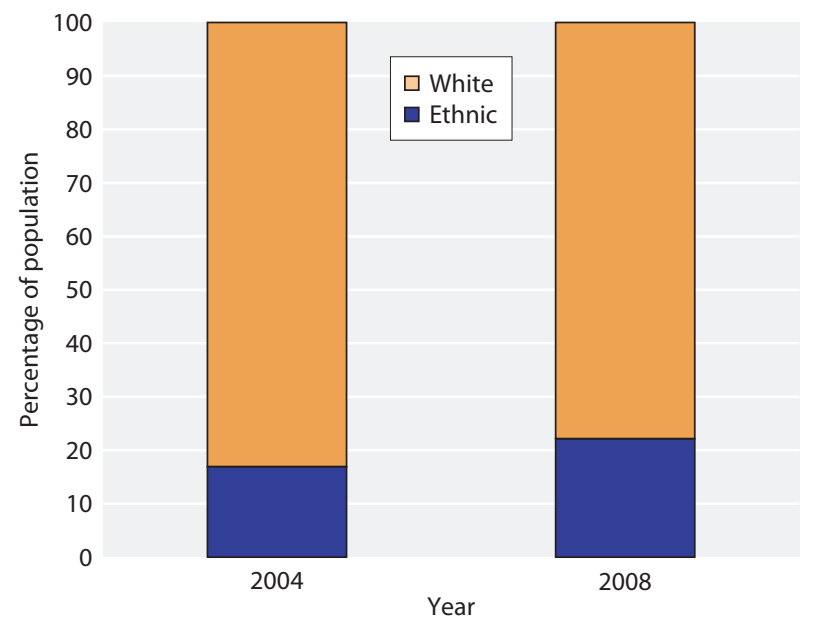

Fig. 13.2. The proportions of prevalent paediatric RRT patients in 2004 and 2008 from ethnic minorities

\section{Incidence and prevalence}

The incidence and prevalence of ERF in the UK has been calculated using estimated population figures for the UK from the Office for National Statistics online resource [1]. The overall prevalence of ERF in children under the age of 16 years in the UK was 55 per million age related population. The prevalence was highest at $96.3 \mathrm{pmp}$ in the 12 to 16 year age group. At all ages there was a significant excess of males (table 13.4), which was similar to that found in the adult population. The adult UK Renal Registry is recording additional data on a prevalence rate of $50 \mathrm{pmp}$ in patients aged 16-19 which would potentially increase this rate to $117 \mathrm{pmp}$. This contrasts with a rate of $230 \mathrm{pmp}$ seen in the $20-24$ year age band.

The incidence of ERF (or take on rate of new patients) is shown in table 13.5. Whilst there was quite wide year to year variability in some age bands, the degree of variation in the total under 16 year old cohort was less. The only trend with time seemed to be a decrease in the incidence of ERF in patients between 4 and 8 years. This is visually apparent in figure 13.3.

Whilst the prevalence of ERF rose steadily with age, through continued acceptance onto the programme of new patients and survival of existing patients, the distribution of incidence with age showed a V shaped

Table 13.3. ERF prevalent paediatric population in 4 year age bands

\begin{tabular}{lrrrrr}
\hline & \multicolumn{5}{c}{ Patient population for the years of } \\
\cline { 2 - 6 } Age group (yrs) & 2002 & 2003 & 2004 & 2005 & 2008 \\
\hline $0-3.9$ & 49 & 39 & 41 & 36 & 69 \\
$4-7.9$ & 94 & 103 & 112 & 108 & 92 \\
$8-11.9$ & 185 & 176 & 173 & 152 & 180 \\
$12-15.9$ & 294 & 291 & 297 & 321 & 292 \\
$16-19.9$ & 171 & 164 & 179 & 151 & 218 \\
\hline
\end{tabular}


Table 13.4. Prevalence of ERF per million population by age and gender

\begin{tabular}{lcccccccc}
\hline & \multicolumn{2}{c}{ All patients } & & \multicolumn{2}{c}{ Male } & & \multicolumn{2}{c}{ Female } \\
\cline { 2 - 3 } \cline { 7 - 8 } Age group (yrs) & Patients & Prevalence & & Patients & Prevalence & & Patients & Prevalence \\
\hline $0-3.9$ & 69 & 23.7 & & 44 & 29.5 & & 25 & 17.6 \\
$4-7.9$ & 92 & 34.2 & & 62 & 45.0 & & 29 & 22.1 \\
$8-11.9$ & 180 & 62.6 & & 104 & 70.7 & & 76 & 54.1 \\
$12-15.9$ & 292 & 96.3 & & 177 & 113.8 & & 114 & 77.3 \\
$16-19.9$ & 218 & 67.5 & & 127 & 76.3 & & 90 & 57.6 \\
$<15$ & 536 & 50.0 & & 327 & 59.6 & & 207 & 39.6 \\
$<16$ & 633 & 55.0 & & 387 & 65.7 & & 244 & 43.5 \\
\hline
\end{tabular}

*gender unknown for 3 patients

Table 13.5. Incidence of ERF per million age related population for the last ten years

\begin{tabular}{lrrrrrrrrrrr}
\hline & \multicolumn{10}{c}{ Take on rate per million age related population } \\
\cline { 2 - 12 } Age group (yrs) & 1998 & 1999 & 2000 & 2001 & 2002 & 2003 & 2004 & 2005 & 2006 & 2007 & Mean \\
\hline $0-3.9$ & 8.6 & 6.2 & 9.3 & 8.3 & 9.6 & 5.5 & 6.2 & 5.2 & 6.9 & 8.9 & 7.5 \\
$4-7.9$ & 5.9 & 4.5 & 5.9 & 5.6 & 6.7 & 6.7 & 3.0 & 4.1 & 4.1 & 2.6 & 4.9 \\
$8-11.9$ & 8.7 & 8.0 & 10.1 & 10.9 & 9.4 & 8.7 & 3.1 & 2.8 & 9.4 & 4.9 & 7.6 \\
$12-15.9$ & 13.9 & 12.9 & 7.9 & 13.9 & 14.2 & 13.2 & 11.9 & 7.9 & 6.9 & 11.6 & 11.4 \\
$16-19.9$ & 1.9 & 3.1 & 5.3 & 4.0 & 1.6 & 2.8 & 2.2 & 1.6 & 1.6 & 2.2 & 2.6 \\
$<15$ & 9.2 & 8.0 & 8.4 & 9.4 & 9.9 & 8.3 & 6.1 & 4.7 & 6.6 & 6.9 & 7.8 \\
$<16$ & 9.4 & 8.0 & 8.3 & 9.7 & 10.1 & 8.6 & 6.2 & 5.0 & 6.9 & 7.1 & 7.9 \\
\hline
\end{tabular}

curve with the incidence in the first and third 4 year blocks being similar, a nadir between 4 and 8 years and then a peak at 12 to 16 years. This is demonstrated in figure 13.4.

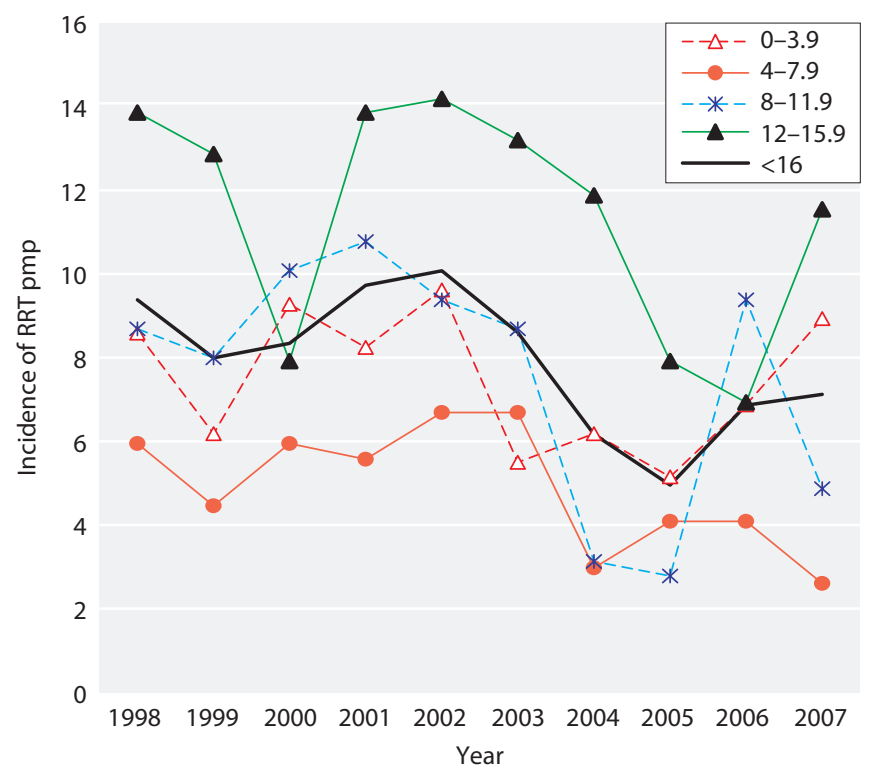

Fig. 13.3. Paediatric incidence of RRT pmp 1998-2007 by age at onset
Both the incidence and prevalence of ERF varied with ethnicity. The South Asian population (patients from the Indian subcontinent) showed a prevalence 2.7 times that of the White population. The incidence of ERF in this group averaged out at 2.5 times that of the White population over the past 10 years. The prevalence and incidence of ERF in the Black population was just slightly

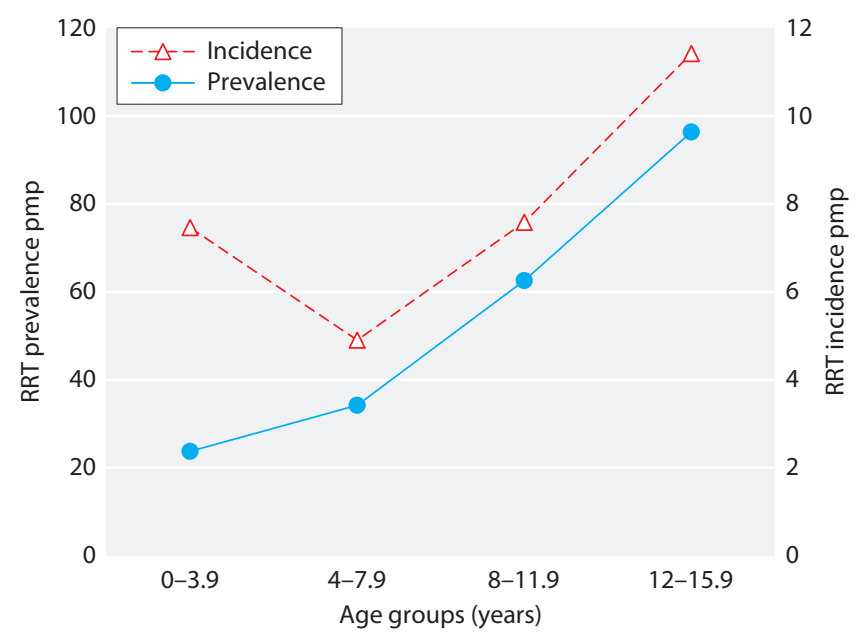

Fig. 13.4. Incidence and prevalence of RRT pmp in 2008 by age
Lewis/Shaw/Sinha/Adalat/Hussain/ Inward 


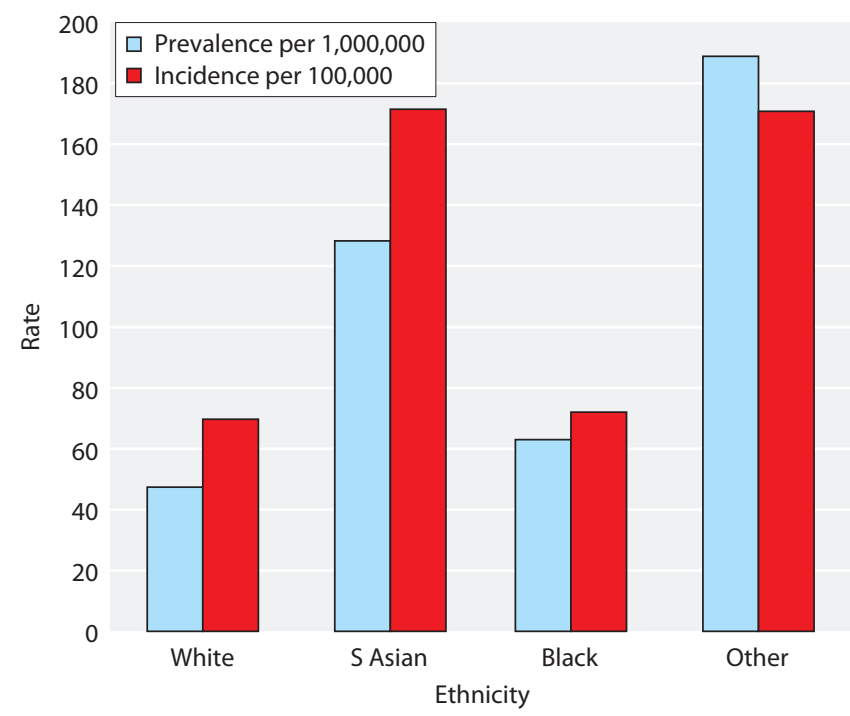

Fig. 13.5. Incidence per 100,000 age related population and prevalence pmp by ethnicity

higher than that of the White population. Those classified as 'Other' had a prevalence 4 times that of the White population and an incidence similar to that of the South Asian population (figure 13.5).

\section{Causes of ERF}

The causes of ERF in the paediatric population have been previously outlined [2]. The number of individual diseases and sub classifications are numerous. For analytical purposes these are best broken down into a smaller number of disease categories. Table 13.6 shows these disease categories for 744 of the 875 current patients $(85 \%)$ for whom a causative diagnosis was listed. Renal dysplasia with or without vesico-ureteric reflux was the predominant cause accounting for one third of all patients. The combination of glomerulonephritic diseases and obstructive uropathy accounted for another third and the final third was composed of the other 8 categories. The male:female ratio for patients with renal dysplasia was high and this, together with the vast excess of males with obstructive uropathy from posterior urethral valves, accounted for the overall predominance of males in the population.

When examining the role of ethnicity in the distribution of causes of ERF the proportion of patients from ethnic minority groups varied widely between categories. This is demonstrated in table 13.7. The highest proportion of ethnic minority patients was in the group with an unknown aetiology, these being patients who have presented at or near established renal failure in whom it was not possible to elucidate the underlying cause. At $7.9 \mathrm{pmp}$, the prevalence of patients with renal failure of unknown aetiology in ethnic minority patients was almost 10 times that of White patients. This excess proportion of ethnic minority patients with no known underlying cause was significant $(p=0.0003)$. Renal dysplastic conditions were significantly under-represented in the ethnic minority groups $(\mathrm{p}=0.0074)$. However, this was only because of the higher prevalence of other conditions. The prevalence of renal dysplasia in ethnic minority patients overall at $23.2 \mathrm{pmp}$ is 1.4 times that of the White population. For the South Asian population the prevalence of tubulointerstitial diseases and congenital nephrosis were much higher than in the White or Black populations. Some of the differences in disease frequencies related to disease inheritance. Table 13.8 shows the numbers and prevalence of patients with recognised inherited disease according to ethnicity. There was a significant increase in the proportion of patients with autosomal recessively

Table 13.6. Diagnostic groups and gender distribution of the prevalent paediatric ERF population

\begin{tabular}{|c|c|c|c|c|c|}
\hline Diagnostic group & Patients & Proportion of total (\%) & Male & Female & $\mathrm{M}: \mathrm{F}$ ratio \\
\hline Renal dysplasia \pm reflux & 251 & 33.7 & 164 & 87 & 1.9 \\
\hline Glomerular diseases & 129 & 17.3 & 60 & 69 & 0.9 \\
\hline Tubulo-interstitial disease & 58 & 7.8 & 26 & 32 & 0.8 \\
\hline Congenital nephrosis & 55 & 7.4 & 22 & 33 & 0.7 \\
\hline Metabolic diseases & 31 & 4.2 & 13 & 18 & 0.7 \\
\hline Unknown aetiology & 24 & 3.2 & 8 & 16 & 0.5 \\
\hline Malignancy & 14 & 1.9 & 6 & 8 & 0.8 \\
\hline Drug nephrotoxicity & 6 & 0.8 & 3 & 3 & 1.0 \\
\hline
\end{tabular}


Table 13.7. Diagnostic groups and ethnic distribution of the prevalent ERF paediatric population

\begin{tabular}{|c|c|c|c|c|c|c|c|c|c|}
\hline \multirow{2}{*}{ Diagnostic group } & \multicolumn{8}{|c|}{ Ethnicity } & \multirow{2}{*}{$\begin{array}{c}\text { Percentage } \\
\text { ethnic } \\
\text { minority }\end{array}$} \\
\hline & \multicolumn{2}{|c|}{ White } & \multicolumn{2}{|c|}{ S Asian } & \multicolumn{2}{|c|}{ Black } & \multicolumn{2}{|c|}{ Other } & \\
\hline Renal dysplasia \pm reflux & 213 & 16.3 & 26 & 24.3 & 6 & 14.1 & 6 & 42.3 & 15.1 \\
\hline Glomerular diseases & 96 & 7.3 & 28 & 26.2 & 5 & 11.7 & 0 & 0.0 & 25.6 \\
\hline Congenital nephrosis & 39 & 3.0 & 16 & 15.0 & 0 & 0.0 & 0 & 0.0 & 29.1 \\
\hline Metabolic diseases & 24 & 1.8 & 5 & 4.7 & 0 & 0.0 & 2 & 14.1 & 22.6 \\
\hline Renovascular disease & 29 & 2.2 & 1 & 0.9 & 1 & 2.3 & 0 & 0.0 & 6.5 \\
\hline Polycystic kidney disease & 20 & 1.5 & 2 & 1.9 & 1 & 2.3 & 1 & 7.0 & 16.7 \\
\hline Unknown aetiology & 11 & 0.8 & 9 & 8.4 & 2 & 4.7 & 2 & 14.1 & 54.2 \\
\hline Malignancy & 12 & 0.9 & 1 & 0.9 & 1 & 2.3 & 0 & 0.0 & 14.3 \\
\hline
\end{tabular}

inherited diseases in patients from ethnic minority groups ( $\mathrm{p}=0.0087)$.

To establish whether there was any change in the pattern of disease causing ERF with time the distribution of diagnostic groups in patients between the ages of 0 and 15 years was examined. This was then compared to the distribution of diagnostic groups in a cohort of registered patients whose age would now be between 25 and 40 years. There were 460 current patients under the age of 15 years with diagnoses stated. In addition there were 58 deceased patients, 10 patients transferred to non-UK centres and 17 patients whose whereabouts and outcome were unknown who were registered and had a diagnosis. This gave an under 15 years of age cohort of 545, of whom 330 were male and 215 were female (M:F 1.53). There were 299 patients whose details were on the Paediatric Registry, including a causative diagnosis and who would have been between 25 and 40 years of age at the audit point of 1st April 2008. Males comprised
186 of this cohort (M:F 1.64:1). The distribution of the diagnostic groups is given in table 13.9.

Whilst the proportion of patients with renal dysplasia and/or reflux nephropathy was the same between the two groups it is interesting to note that in the under 15 years of age group just $15(8 \%)$ were primarily categorised as having reflux nephropathy whilst 58 of the 104 patients $(56 \%)$ in the $15-40$ year old age group were classified as having reflux nephropathy $(\mathrm{p}<0.0001)$. Similarly, whilst posterior urethral valves is the predominant cause of ERF in both groups a significantly higher proportion of those in the younger cohort (85\%) with obstructive uropathy had this as a cause when compared with $62 \%$ in the older cohort $(\mathrm{p}=0.0015)$. In parallel with this there has been a small but significant reduction in the proportion of patients with neuropathic bladder as a cause of ERF in the younger cohort (5\% vs $16 \%, \mathrm{p}=0.0435$ ).

Glomerulonephritis leading to ERF was significantly more common in the older cohort of patients

Table 13.8. Inherited diseases and ethnicity in the prevalent ERF paediatric population

\begin{tabular}{|c|c|c|c|c|c|c|c|c|}
\hline \multirow{2}{*}{ Inheritance } & \multicolumn{8}{|c|}{ Ethnicity } \\
\hline & \multicolumn{2}{|c|}{ White } & \multicolumn{2}{|c|}{ S Asian } & \multicolumn{2}{|c|}{ Black } & \multicolumn{2}{|c|}{ Other } \\
\hline Autosomal recessive & 112 & 8.5 & 32 & 30.0 & 1 & 2.3 & 6 & 42.3 \\
\hline Autosomal dominant & 7 & 0.5 & 0 & 0.0 & 0 & 0.0 & 0 & 0.0 \\
\hline None or other or undefined & 458 & 35.0 & 85 & 79.7 & 18 & 42.2 & 12 & 84.5 \\
\hline
\end{tabular}


Table 13.9. Distribution of diagnostic groups for patients with ERF in childhood who were aged $<15$ yrs on $1 / 4 / 2008$, compared with those who would have been between 15-40 years of age on 1/4/2008

\begin{tabular}{lcccc}
\hline Diagnostic group & Patients $<15$ yrs & Proportion of total $\%$ & Patients $25-40$ yrs & Proportion of total \% \\
\hline Renal dysplasia \pm reflux & 187 & 34.3 & 104 & 58 \\
Obstructive uropathy & 95 & 17.4 & 73 & 34.8 \\
Glomerular diseases & 77 & 14.1 & 23 & 24 \\
Tubulo-interstitial disease & 31 & 5.7 & 4 & 7.7 \\
Metabolic diseases & 16 & 2.9 & 4 & 8.0 \\
Congenital nephrosis & 52 & 9.5 & 2 & 1.3 \\
Polycystic kidney disease & 24 & 4.4 & 2 & 1.3 \\
Renovascular disease & 33 & 6.1 & 2.6 & 0.7 \\
Malignancy & 14 & 0.6 & 4 & 0.3 \\
Drug nephrotoxicity & 3 & 2.4 & & 1.3 \\
Unknown aetiology & 13 & &
\end{tabular}

$(\mathrm{p}=0.0002)$. Similarly metabolic diseases causing ERF were more common in the older cohort $(\mathrm{p}=0.0019)$. The single most common metabolic disease causing ERF in childhood was nephropathic cystinosis. Of the 545 patients in the currently under 15 years of age group, 7 had cystinosis. Of the 299 patients now between 15 and 40 years of age, 21 had cystinosis. Thus the incidence of cystinosis causing ERF in childhood has significantly reduced over a generation $(\mathrm{p}<0.0001)$. In contrast the number and proportion of patients with congenital nephrosis, polycystic kidney disease and renovascular disease (mainly cortical necrosis) were all significantly higher in the younger aged cohort (congenital nephrosis $\mathrm{p}<0.0001$, polycystic disease $\mathrm{p}=0.016$, renovascular disease $\mathrm{p}<0.0001$ ).
There was a difference in ethnicity between the two cohorts. The proportion of ethnic minority patients in the younger cohort was $22.4 \%$ as one would expect from the current ERF population statistics whilst the proportion of ethnic minority patients in the older cohort was $18.1 \%(\mathrm{p}=0.0139)$.

\section{Survival}

To assess 5 year survival (and hence mortality) data were extracted from the Registry on patients starting ERF treatment between 1st April 1998 and 1st April 2003. For each patient the subsequent five year period was analysed to determine the 5 year survival. These data are presented in table 13.10. Patients were divided according to 4 year age bands and year of commencement of treatment. It

Table 13.10. Mortality over the first 5 years after starting RRT in the paediatric population

\begin{tabular}{|c|c|c|c|c|c|c|c|c|c|}
\hline \multirow[b]{3}{*}{ Age (yrs) } & \multicolumn{9}{|c|}{ Year of commencement of ERF treatment } \\
\hline & \multicolumn{3}{|c|}{1998} & \multicolumn{3}{|c|}{1999} & \multicolumn{3}{|c|}{2000} \\
\hline & Patients & Deaths & Mortality & Patients & Deaths & Mortality & Patients & Deaths & Mortality \\
\hline $0-3.9$ & 25 & 4 & $16.0 \%$ & 18 & 5 & $27.8 \%$ & 27 & 5 & $18.5 \%$ \\
\hline $4-7.9$ & 16 & 1 & $6.3 \%$ & 12 & 0 & $0.0 \%$ & 16 & 0 & $0.0 \%$ \\
\hline $8-11.9$ & 25 & 2 & $8.0 \%$ & 23 & 0 & $0.0 \%$ & 29 & 1 & $3.5 \%$ \\
\hline $12-15.9$ & 42 & 1 & $2.4 \%$ & 39 & 1 & $2.6 \%$ & 24 & 1 & $4.2 \%$ \\
\hline \multirow[t]{2}{*}{$<16$} & 108 & 8 & $7.4 \%$ & 92 & 7 & $7.6 \%$ & 96 & 7 & $7.3 \%$ \\
\hline & \multicolumn{3}{|c|}{2001} & \multicolumn{3}{|c|}{2002} & \multicolumn{3}{|c|}{ Total 1998-2002 } \\
\hline Age (yrs) & Patients & Deaths & Mortality & Patients & Deaths & Mortality & Patients & Deaths & Mortality \\
\hline $0-3.9$ & 24 & 3 & $12.5 \%$ & 28 & 9 & $32.1 \%$ & 122 & 26 & $21.3 \%$ \\
\hline $4-7.9$ & 15 & 2 & $13.3 \%$ & 18 & 2 & $11.1 \%$ & 77 & 5 & $6.5 \%$ \\
\hline $8-11.9$ & 31 & 1 & $3.2 \%$ & 27 & 1 & $3.7 \%$ & 135 & 5 & $3.7 \%$ \\
\hline $12-15.9$ & 42 & 3 & $7.1 \%$ & 43 & 0 & $0.0 \%$ & 190 & 6 & $3.2 \%$ \\
\hline$<16$ & 112 & 9 & $8.0 \%$ & 116 & 12 & $10.3 \%$ & 524 & 43 & $8.2 \%$ \\
\hline
\end{tabular}


should be noted that not all those over the age of 12 years when starting treatment after 2000 will have had a full 5 year follow up as some will have been transferred to adult centres and deaths may not have been reported back. The overall 5 year survival rate for children starting ERF treatment below the age of 16 years was $91.2 \%$. Mortality was highest for those starting ERF treatment in the first 4 years of life and then declined. Most fatalities occur within the first 36 months of starting treatment but later fatalities also occur and some of those surviving 5 years subsequently died. These data do not take account of patients who, for one reason or another, had not been started on a renal replacement therapy regimen.

Although mortality in those starting RRT between the ages of 4 and 8 years was higher than that for patients starting between 8 and 12 years, this difference did not reach statistical significance. Mortality for those starting RRT within the first four years of life was significantly greater than those starting RRT in the subsequent 8 years of life $(\mathrm{p}<0.0001)$. Looking at this group in more detail it was clear that the mortality was highest for those starting dialysis in the first year of life and then declined as the age of treatment commencement rose (figure 13.6).

\section{Current modality of RRT}

Of the 875 current patients, some details of treatment modality were available for 847 (96.8\%). Of these, 629 $(74.3 \%)$ had a functioning renal allograft. Peritoneal dialysis was the active modality in $123(14.5 \%)$ and haemodialysis was being used in 92 (10.9\%). Three patients were on no active treatment at the time of audit $(0.4 \%)$, two of these were managing on their

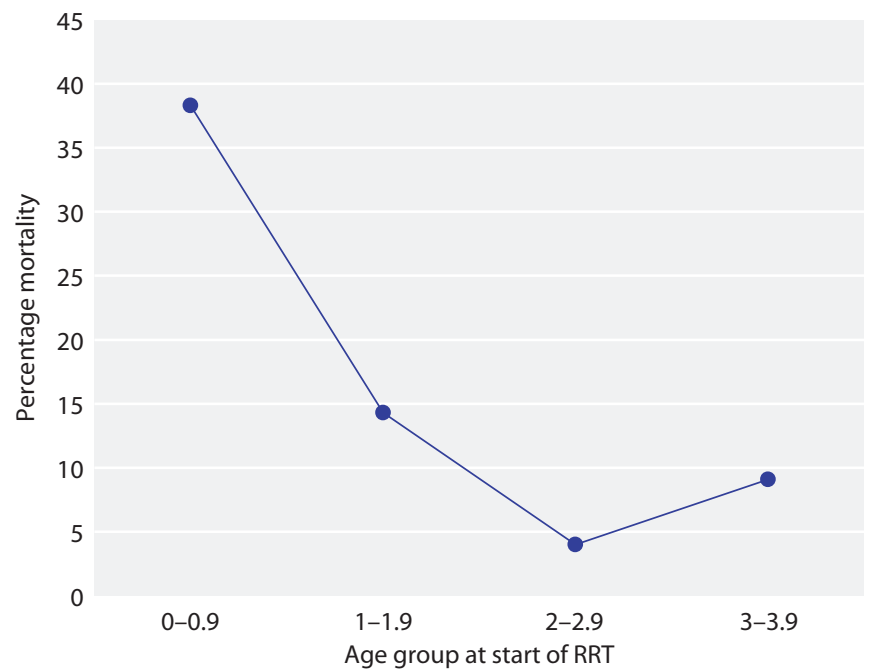

Fig. 13.6. Mortality within 5 years of starting RRT by age at start of RRT

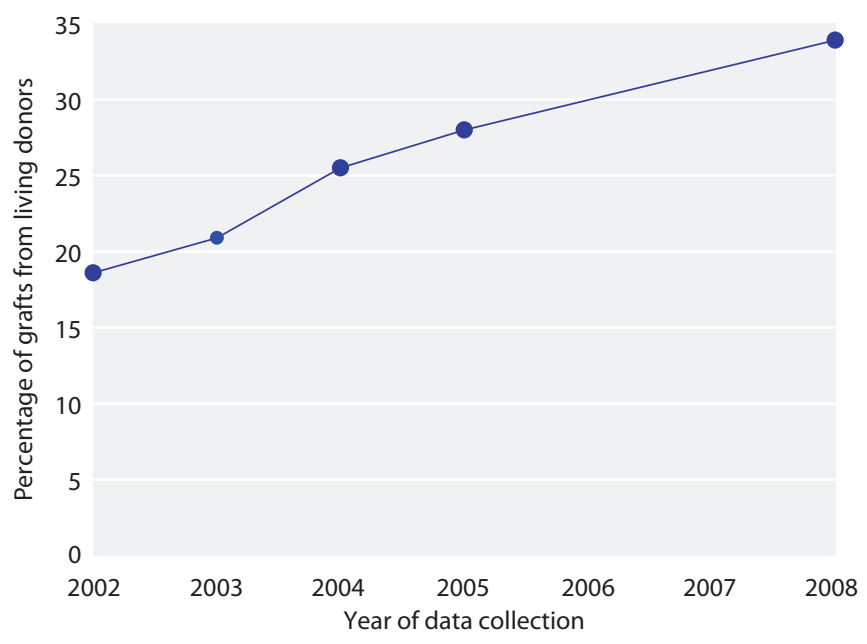

Fig. 13.7. Percentage of prevalent paediatric renal transplant patients with a living donor graft, by year

residual renal function at the time having previously been on dialysis and one patient was no longer receiving treatment after discussion with the family. This patient had a heavy burden of comorbidity and disability.

Of the 629 patients with transplants, the type of allograft was known in 614 (97.6\%). Living donation (LD) accounted for 208 grafts (33.9\%) and $406(66.1 \%)$ were from deceased donors (DD). The proportion of paediatric patients with allografts from living donors has been steadily increasing as demonstrated in figure 13.7.

Figure 13.8 shows the distribution of LD grafts and DD grafts in different ages of children. A much greater proportion of young children have an LD graft than older children.

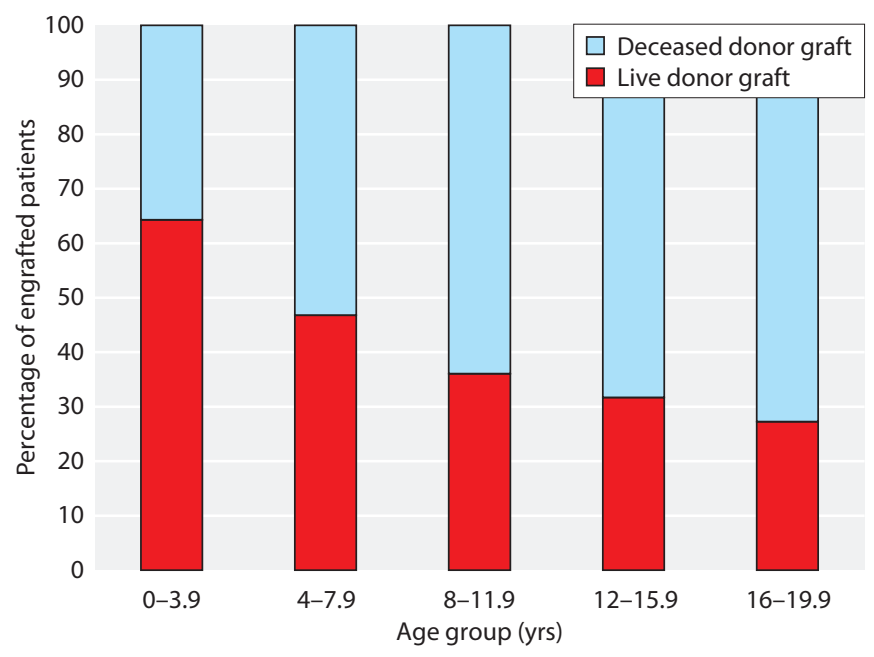

Fig. 13.8. Percentage of engrafted paediatric patients with an LD graft or DD graft by age 


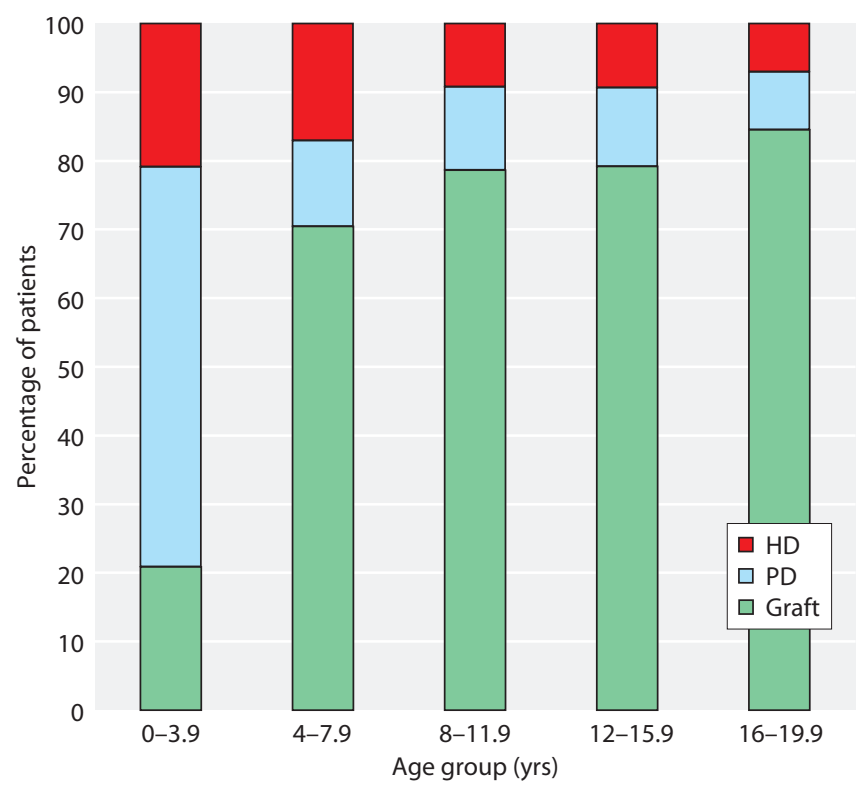

Fig. 13.9. Distribution of RRT modalities by age

For those on dialysis, $42.8 \%$ were having haemodialysis. For those having peritoneal dialysis, the vast majority $(85.4 \%)$ were being treated with automated peritoneal dialysis (APD), the remainder being on CAPD. Figure 13.9 shows the distribution of all modalities according to age. Only $20 \%$ of patients in the first 4 years of life had an allograft. This figure rapidly rose to about $80 \%$ in the 8 to 12 year old group and remained at this level thereafter. Beyond the age of 4 years those on dialysis were fairly evenly split between peritoneal dialysis and haemodialysis, whilst peritoneal dialysis predominated in the first 4 years of life.

The distribution of treatment modalities was different between the White patients and those from ethnic minority groups. A significantly larger proportion of White patients had been transplanted than ethnic minority patients $(p=0.0003)$. For those who had been engrafted, $36 \%$ of White patients had an LD graft compared to $23 \%$ of ethnic minority patients $(p=0.0116)$. For those on dialysis, $50 \%$ of those from ethnic minority groups were on haemodialysis compared to $38 \%$ of White patients $(\mathrm{p}=\mathrm{ns})$. These data are demonstrated in figure 13.10.

\section{Discussion}

ERF paediatric population, incidence and prevalence

Taking into account variation secondary to the completeness of data collection, it is apparent that there was

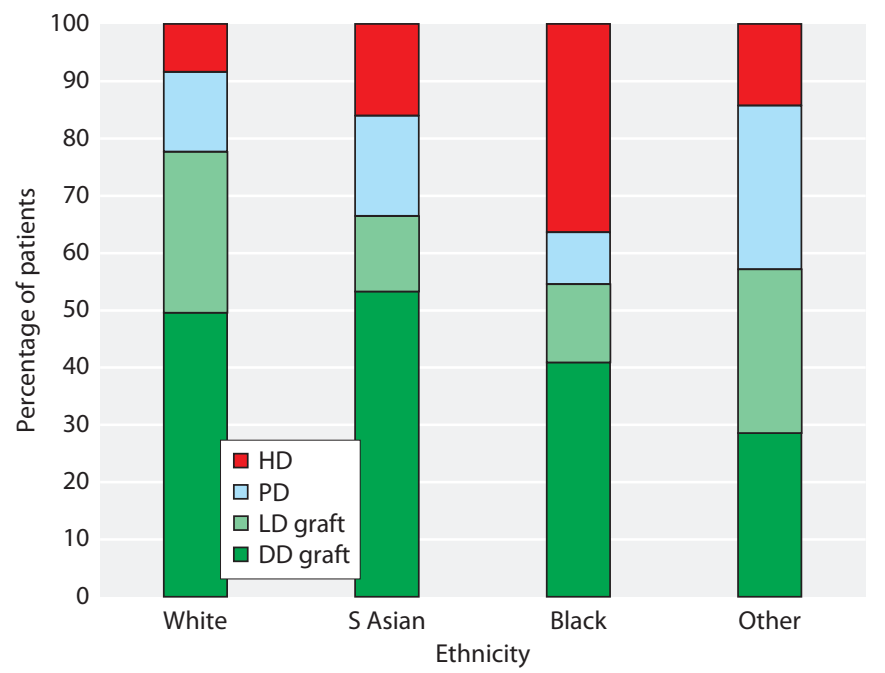

Fig. 13.10. RRT modality by ethnicity

only a slow incremental growth of the paediatric ERF population with time. The incidence and prevalence rates of childhood ERF have not changed significantly from our previous report [2] and were similar to those quoted in the ANZDATA 2007 Registry Report [3]. The increase in the number of patients below 4 years of age in the current data was unlikely to be representative of a change in the population. There was significant year to year variability in the incidence of ERF in this age band because of the small numbers involved and looking at incidence over the past 10 years in table 13.5, there was no clear trend. Comparing incidence rates to previously published rates for both European and non European countries [4], the current UK rates are within the ranges described which vary according to predisposition to particular diseases according to ethnicity and healthcare provision.

Whilst the overall incidence and prevalence have not changed significantly the distribution of the population between those who are White and those from ethnic minority groups has. This was to be expected with the high incidence in these ethnic minority groups. Whilst the largest ethnic minority group of South Asian patients has always shown an incidence about three times that of the White population, the 'Other' group has for the first time in this report shown an even higher incidence. This relates to the fact that the majority of these patients come from an ethnic background where consanguineous marriage is common.

\section{Causes of ERF}

Renal dysplasia and related conditions were the most common cause of ERF, accounting for one third of all 
patients, in the current population. Glomerular diseases and obstructive uropathy together accounted for a further third. This data appears to be different to our previous report [2] where renal dysplasia accounted for just over $24 \%$ of cases and glomerular diseases $22 \%$ of cases. In reality however, there has been no change in the causative disease pattern in the population. The analysis in this report looked at the causative diseases in the current population. Our previous report looked at a larger cohort who had presented after 1996. This larger cohort included a number of patients who had been transferred to adult services. As renal dysplasia is a cause of ERF presenting at all ages, but glomerular diseases tend to just present in mid and late childhood the length of time patients with ERF from glomerular disorders spend in paediatric departments is less than that for patients with renal dysplasia. Thus both analyses are correct. Looking in a cross-sectional manner at any time point, a third of children with ERF will have renal dysplasia as a cause. Looking at the number of children passing through paediatric services the proportions with renal dysplasia and glomerular disorders were not dissimilar.

Ethnicity played a significant role in the distribution of diseases causing ERF. A significantly lower proportion of ethnic minority patients had renal dysplasia even though the prevalence was higher than in the White population. This was because diseases associated with autosomal recessive inheritance are significantly more common with a markedly higher prevalence. As the proportion of the ERF population composed of ethnic minority groups increases with time it is likely that the pattern of causative diseases will change. It is noteworthy that 54\% of patients who presented late with no definable diagnosis were from ethnic minority groups. This would suggest that there is reduced awareness of problems and delayed contact with healthcare services in these groups.

Examining the distribution of causes of renal failure in the current generation of patients under 15 years of age and comparing it to a generation now between 25 and 40 years of age, showed a number of interesting points. There has been little change in the proportions of the population with renal dysplasia and obstructive uropathy as a cause. However, whilst just $8 \%$ of the younger cohort have been classified as having reflux nephropathy, 54\% of the older cohort were classified in this way. This could simply be due to a change in the way paediatric nephrologists classify patients presenting with small kidneys and vesico-ureteric reflux. Further analysis of the spectrum of age at presentation, age at commencement of ERF and presence or absence of recurrent urinary sepsis would help define whether there has or has not been a change in the diseases seen. For those with obstructive uropathy the proportion with posterior urethral valves as a cause was significantly higher in the younger cohort. This is likely to represent improved management of neuropathic bladder and acquired obstructive uropathy in the current population together with the survival and acceptance onto RRT programmes of infants with posterior urethral valves who would previously have died.

Glomerular disease was significantly more common as a cause of ERF in the older cohort than the younger generation. There was no evidence to suggest that the incidence of glomerulonephritis has reduced with time. This could suggest that treatment at an early stage for glomerular diseases has improved and fewer are progressing to ERF. Whilst this is possible, this cannot be proven from the data available here. Moreover, the acceptance of infants and very young children onto an RRT programme was not the norm for the generation of the older cohort. This means that they will have had a higher mean age of onset of ERF, and as discussed above, the proportion of patients with glomerular diseases as a cause of ERF in those presenting in later childhood is increased. Thus, whilst more analysis of this phenomenon is required, it is most likely that the decreased proportion of patients with glomerular diseases in the younger cohort is a feature of the distribution of the population rather than an effect of improved treatment for glomerulonephritis.

Contrary to this, metabolic diseases and particularly cystinosis are significantly less common in the younger cohort. There was no evidence to suggest that the incidence of cystinosis is decreasing, indeed, as it is a common disease in communities with a high rate of consanguinity, one might expect it to be increasing [5]. Thus the fall in the numbers of ERF patients with cystinosis between cohorts is likely to be representative of improved diagnosis and treatment for the condition.

Conditions such as congenital nephrosis, recessive polycystic kidney disease and cortical necrosis were significantly under-represented in the older cohort. This was because infants and young children were generally not being accepted onto ERF programmes at that time.

\section{Survival}

Little is written about survival for paediatric patients on an ERF programme. This report shows an overall 5 year survival for patients under the age of 16 of $91.8 \%$. As patients starting dialysis over the age of 12 years 
could have been transferred to adult centres and died before the five year point and without being registered as deceased this could be a slight overestimate of survival. However, just looking at patients starting RRT below the age of 12 years, 5 year survival is $89.3 \%$. This figure is not dissimilar to those quoted by USRDS [6], though direct comparison is not possible as the USRDS figures are quoted as probabilities by RRT modalities. The highest mortality was in the youngest patients, with almost $40 \%$ of patients commencing treatment within the first year of life dying. As described by Wood et al. [7], much of the mortality in this group will have been related to comorbidities. The $83-89 \%$ survival rate for patients starting dialysis in the first year of life described by Wood et al. seems much better than in this report. However, it should be noted that they reviewed one rather than 5 year survival and more importantly, it was not a true cohort study but a selected population. Indeed the survival of infants with ERF in the UK may actually be worse than reported here if there were infants in whom a decision not to embark upon ERF treatment was made and these children were not reported to the Paediatric Registry. Further analysis of these data looking at treatment modality and comorbidities in detail is indicated, possibly with a prospective two year data collection of all infants with ERF (whether or not they were accepted onto a treatment programme) to allow a true prognosis to be given to the families of children presenting with ERF in infancy.

\section{Current RRT modality}

The $74.3 \%$ of patients whose current RRT modality was a functioning renal allograft was slightly higher than the $71 \%$ reported by both ANZDATA [2] and the USRDS [6]. This figure has remained stable over the years of data collection by the Registry. For those without an allograft, peritoneal dialysis remained the most prevalent treatment though the percentage of patients receiving haemodialysis had risen to $43 \%$. This is in keeping with the general trend towards increasing haemodialysis therapy in children described by Warady [8].

The proportion of patients with a graft from a living donor continued to rise indicating a continuing preference for living donation. The steadily decreasing proportion of grafts coming from living donors as patient age increases simply represents the past preference for deceased donor transplantation and the continued survival of these grafts in patients who were young at the time of engraftment.

Patients from ethnic minority groups were significantly more likely to be on dialysis than White patients. This was related to the higher prevalence of blood group $B$ in this population and the different distribution of HLA tissue types, making the chance of getting a good match from the predominantly White deceased donor pool poor. As morbidity and mortality are higher in dialysis compared to engrafted patients [8], an education programme promoting living donation in the ethnic minority population is needed. Live donation from ethnic minorities may remain more difficult than Whites, due to a much higher incidence of CKD and renal failure seen in the adult ethnic minorities when compared to Whites.

Conflict of interest: none

\section{References}

1 UK National Statistics Office. http://www. statistics.gov.uk

2 Lewis M., Shaw J. et al. Demography and management of childhood established renal failure in the UK (chapter 13). Nephrology Dialysis Transplantation 2007. 22 Suppl 7:vii165-75.

3 McTaggart S., McDonald S., Henning P., and Dent H. Chapter 11 Paediatric Report. ANZDATA Registry Report 2008, Australia and New Zealand Dialysis and Transplant Registry, Adelaide, South Australia.
4 Stewart J. H., McCredie M. R. et al. Geographic, ethnic, age-related and temporal variation in the incidence of end-stage renal disease in Europe, Canada and the Asia-Pacific region, 1998-2002. Nephrol Dial Transplant 2006. 21(8):2178-83.

5 Madani K., Otoukesh H. et al. Chronic renal failure in Iranian children. Pediatr Nephrol 2001. 16(2):140-4.

6 U.S. Renal Data System, USRDS 2008 Annual Report: Atlas of End-stage Renal Disease in the United States. National Institute of Health, National Institute of Diabetes and Digestive and Kidney Diseases, Bethesda, MD, 2008.

7 Wood E. G., Hand M. et al. Risk factors for mortality in infants and young children on dialysis. Am. J. Kidney Dis. 2001. 37(3);573-9.

8 Warady B. A. and Chadha V. Chronic kidney disease in children: the global perspective. Pediatr Nephrol 2007. 22(12):1999-2009. 
